\title{
Pesquisa Apreciativa: \\ características, utilização e possibilidades para a área da Saúde no Brasil
}

Cristiane Trivisiol Arnemann (a)

Denise Gastaldo(b)

Maria Henriqueta Luce Kruse ${ }^{(c)}$

Arnemann CT, Gastaldo D, Kruse MHL. Appreciative Inquiry: characteristics, utilization and possibilities for the field of Health in Brazil. Interface (Botucatu). 2018; 22(64):121-31.

In this paper we present and discuss the characteristics of Appreciative Inquiry as a research methodology, utilizing an example from an integrated multi-professional residency program in Health in Brazil. Appreciative Inquiry is a methodology used to identify the best practices developed and utilized by people who work in the same institution. This methodology allows the participation and engagement of Health professionals in studies related to their area of work, potentially able to be used in several areas. In addition, Appreciative Inquiry promotes critical and reflexive debates among participants, establishing a space for the production of change. The Health professionals involved in our study considered relevant the goal of encouraging people to adopt a positive, constructive, and dialogical approach to propose institutional changes.

Keywords: Appreciative Inquiry. Qualitative research. Method. Methodology. Residency program.
Nosso artigo apresenta e discute as características da Pesquisa Apreciativa como metodologia de pesquisa, utilizando um exemplo de uma residência multiprofissional integrada em Saúde no Brasil. A Pesquisa Apreciativa, conhecida em inglês como Appreciative Inquiry, é uma metodologia usada para identificar as melhores práticas desenvolvidas e empregadas pelas pessoas que trabalham em uma instituição. Essa metodologia permite a participação e o engajamento de profissionais da área da Saúde em pesquisas relacionadas à sua área de atuação, com potencial para ser aplicada em múltiplas áreas. Além disso, a Pesquisa Apreciativa incentiva debates reflexivos e críticos por parte dos participantes, estabelecendo um espaço de discussão para que mudanças ocorram. Os profissionais da Saúde que participaram desse trabalho consideram pertinente a finalidade de encorajar as pessoas a adotarem uma abordagem positiva, construtiva e dialógica para propor mudanças institucionais.

Palavras-chave: Pesquisa Apreciativa. Pesquisa qualitativa. Métodos. Metodologia. Prática profissional.

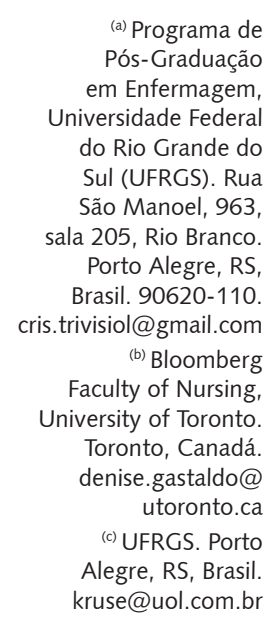




\section{Introdução}

A Pesquisa Apreciativa, também chamada de Investigação Apreciativa por alguns autores, é uma metodologia da mesma família da Pesquisa Ação e da Pesquisa Participativa. Tais metodologias são utilizadas para entender como o conhecimento construído participativamente pode oferecer informações para realizar uma transformação social'. Os pesquisadores que utilizam tais metodologias afirmam que elas são um meio de facilitar o processo reflexivo e permitem obter novos insights a partir da experiência prática dos participantes, desejam compartilhar seu saber e melhorar uma situação de interesse para o grupo ${ }^{2-4}$.

Conhecida na língua inglesa como Appreciative Inquiry, a Pesquisa Apreciativa foi desenvolvida na tese de David Cooperrider, apresentada em 1986, no Departamento de Comportamento Organizacional da Case Western Reserve University, em Cleveland, EUA. A Pesquisa Apreciativa baseia-se em teorias de investigação-ação, mudança organizacional e inovação, utilizando uma perspectiva construcionista social orientada pelo respeito e diálogo para apreciar o que há de melhor em uma instituição $0^{5,6}$.

Assim, a Pesquisa Apreciativa é relevante e original porque privilegia a identificação das melhores práticas desenvolvidas e empregadas pelas pessoas que trabalham em uma instituição, onde essas práticas de sucesso são analisadas tendo em vista a sua difusão. Os problemas identificados são reformulados para serem compreendidos como oportunidades e fontes de informação e inspiração para disseminar e aplicar as melhores práticas ${ }^{7}$.

Em outros países, como o Canadá e a Austrália, a Pesquisa Apreciativa já é utilizada em pesquisas na área da Saúde, sobretudo, na Enfermagem ${ }^{8-10}$. No Brasil, esta metodologia ainda está vinculada às áreas da administração e gestão de pessoas, havendo apenas um estudo teórico, na área da Psicologia, abordando os conceitos centrais, fases, e aplicações da Investigação Apreciativa ${ }^{11}$; outro estudo, na área da Saúde Mental, sobre a implementação de um consultório de rua, o qual menciona que as entrevistas realizadas na pesquisa tiveram inspiração na Pesquisa Apreciativa ${ }^{12}$; e, por último, um estudo teórico que trata da Investigação Apreciativa como uma tecnologia para a pesquisa construcionista na Saúde Coletiva ${ }^{13}$.

Consideramos pertinente que a Pesquisa Apreciativa seja empregada em outras áreas do saber, especialmente na área da Saúde no Brasil, uma vez que essa metodologia valoriza as experiências positivas que membros de um grupo social com uma prática compartilhada têm a oferecer para outros que desenvolvem atividades semelhantes e para melhorar as instituições. A referida metodologia é dialógica e baseada nas capacidades dos participantes, pois valoriza as experiências positivas dos mesmos para construir mudanças nos processos institucionais aos quais estão vinculados ${ }^{14}$.

Diante do que foi apresentado, o objetivo deste artigo é introduzir e discutir as características da Pesquisa Apreciativa como método de pesquisa, utilizando um exemplo em uma residência multiprofissional integrada em Saúde realizada no Brasil.

\section{Pesquisa Apreciativa: origens e utilização}

Em sua tese, Cooperrider ${ }^{5}$ coletou dados relativos à características de sucesso e fracasso de líderes médicos de uma determinada instituição nos Estados Unidos. A partir dessa coleta, ele focouse na exploração dos dados positivos das características desses médicos, pois percebeu que tais dados revelaram como os líderes médicos trabalham mais efetivamente nas suas atividades. Com a participação da orientadora de seu estudo, Dra. Suresh Srivastva, Cooperrider ${ }^{5}$ avaliou o sucesso da metodologia que empregou, e, posteriormente, nomeou-a como Appreciative Inquiry, dada a sua característica principal de apontar elementos positivos e facilitar as mudanças organizacionais nos locais em que é empregada ${ }^{15,16}$.

De acordo com Cooperrider ${ }^{15}$, a partir do diálogo entre os participantes, pode-se mudar e melhorar as práticas de rotina, evidenciando os exemplos positivos relacionados: à atuação profissional, as atitudes individuais ou, ainda, trabalho em grupo em prol da instituição à qual os participantes estão vinculados. Assim, essa metodologia utiliza as capacidades dos participantes e 
os processos organizacionais da instituição para propor inovação, fazendo com que as pessoas se sintam coparticipantes dos processos de mudança institucional ${ }^{5,15}$. Esta mudança de significados $e$ comportamentos nos grupos de trabalho deve-se à produção coletiva das opções e dos caminhos para melhoria nos processos e serviços executados ${ }^{15}$.

Neste tipo de pesquisa, trabalhar em conjunto e criar ações coletivas dentro da equipe são considerados componentes importantes para iniciar a mudança ${ }^{10,15,16}$. O destaque dessa intervenção é que cada grupo de trabalho deve encontrar um aspecto positivo que pode ser utilizado para promover mudanças não apenas para os participantes da pesquisa, mas, também, para as pessoas com as quais interagem na instituição. O foco desta metodologia é identificar as capacidades que podem ser aperfeiçoadas e exploradas utilizando os exemplos positivos das experiências dos participantes, o que difere das abordagens participativas tradicionais baseadas na identificação de problemas a serem solucionados, como, por exemplo, na Pesquisa-ação. A Pesquisa Apreciativa transforma o enfoque, de modo que os pontos fortes descobertos pelos participantes são reconhecidos e apreciados para que mudanças possam ser realizadas ${ }^{17}$.

A Pesquisa Apreciativa é um novo método de pesquisa social que recentemente se destaca nos estudos relacionados aos cuidados da saúde ${ }^{8,9}$. A maioria dos estudos ainda está atrelada à prática clínica da Enfermagem, sendo esta metodologia pouco utilizada nas áreas de Saúde Pública e promoção da saúde ${ }^{18}$. A realização de pesquisas que utilizam tal metodologia tem aumentando significativamente na área da Enfermagem ${ }^{19,20}$, e vem sendo utilizada internacionalmente na área da Saúde (Quadro 1).

Quadro 1. Exemplos de publicações de pesquisas na área da Enfermagem que utilizaram a Pesquisa Apreciativa.

\begin{tabular}{|c|c|c|}
\hline Artigos & Referência & Utilização da Pesquisa Apreciativa \\
\hline 1 & Havens DS 19 & Melhorar a comunicação e a colaboração entre enfermeiros e outros profissionais de saúde \\
\hline 2 & Ruhe $M C^{21}$ & Melhorar a qualidade das práticas de cuidados na atenção primária \\
\hline 3 & Scerri $A^{22}$ & $\begin{array}{l}\text { Aumentar a qualidade do trabalho das enfermeiras hospitalares com os cuidados de pacientes } \\
\text { com demência }\end{array}$ \\
\hline 4 & Jongudomkarn $\mathrm{D}^{23}$ & $\begin{array}{l}\text { Desenvolver um modelo de enfermagem para a prevenção do câncer e outras doenças não } \\
\text { transmissíveis }\end{array}$ \\
\hline 5 & Falk KJ24 & $\begin{array}{l}\text { Utilizar a investigação apreciativa com enfermeiras que trabalham com crianças de pais encar- } \\
\text { cerados }\end{array}$ \\
\hline 6 & Trajkovski $\mathrm{S}^{10}$ & $\begin{array}{l}\text { Relatar a experiência de enfermeiras neonatais e pais que trabalharam de forma colaborativa } \\
\text { para aumentar a família cuidado centrado (FCC) na unidade neonatal }\end{array}$ \\
\hline 7 & Baker $\mathrm{A}^{25}$ & Desenvolver uma rede clínica para doenças hepáticas infantis \\
\hline 8 & Lazic J26 & Explorar e desenvolver as relações de trabalho em um serviço de oncologia pediátrica \\
\hline 9 & Yoon $\mathrm{MN}^{17}$ & Promover cuidados de enfermagem em vias orais do paciente \\
\hline 10 & Shendell-Falik N27 & $\begin{array}{l}\text { Melhorar a segurança do paciente por meio de melhores processos de "handoff"' (transferir o } \\
\text { paciente de um profissional ao outro ou de um hospital ao centro de saúde) paciente. }\end{array}$ \\
\hline 11 & Lind $C^{19}$ & $\begin{array}{l}\text { Obter dados preliminares sobre a aceitabilidade de investigação apreciativa (Al) no desenvol- } \\
\text { vimento de pessoal na área da saúde }\end{array}$ \\
\hline 12 & Bonham $\mathrm{E}^{28}$ & Analisar entrevistas de jovens residentes num centro de detenção juvenil \\
\hline 13 & Knibbs $\mathrm{K}^{29}$ & $\begin{array}{l}\text { Gerar recomendações de políticas para a construção de capacidade de enfermagem em saúde } \\
\text { pública }\end{array}$ \\
\hline
\end{tabular}

\section{Fases da metodologia}

A Pesquisa Apreciativa tem quatro fases que constituem o 'ciclo 4D', denominado assim porque

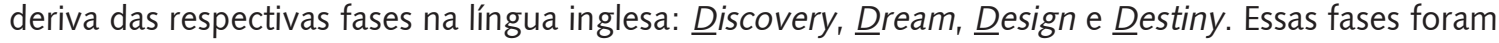
traduzidas para o português como: Descoberta, Sonho, Planejamento e Destino (DSPD; Figura 1). 
Cada fase pode requerer várias reuniões entre os participantes da pesquisa, e os estudos que utilizam esta metodologia podem variar de meses a um ano $0^{9,10}$.

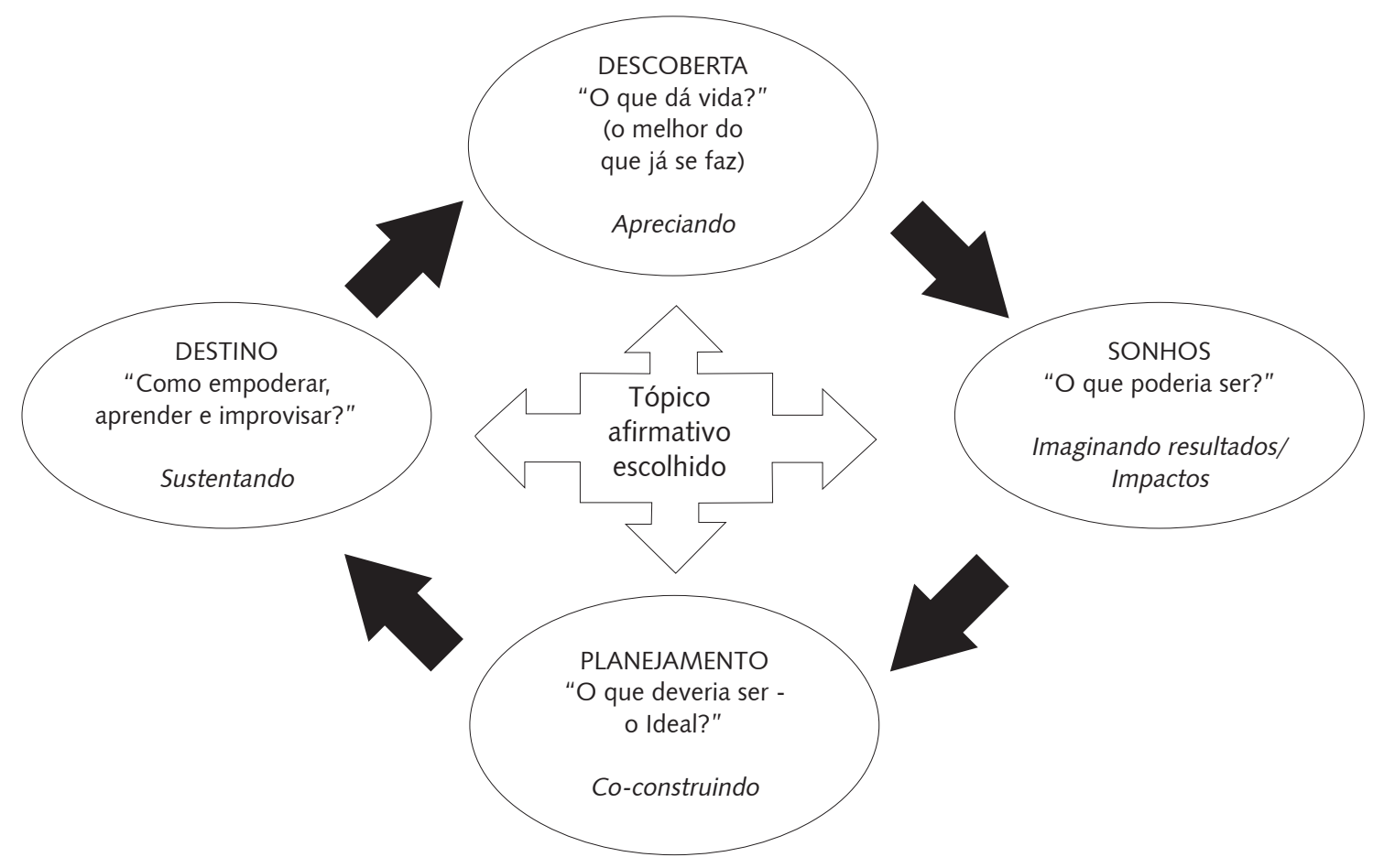

Figura 1. Exemplo da aplicação da metodologia

Nós utilizamos a Pesquisa Apreciativa para conhecermos quais são as melhores práticas que os preceptores desenvolvem, em termos de Educação Permanente em Saúde, em uma residência multiprofissional; como as implementam juntamente com os outros profissionais da residência multiprofissional, e como estas habilidades/capacidades são articuladas em um espaço de formação como a residência multiprofissional.

Em nosso estudo, foram realizadas seis reuniões de, aproximadamente, duas horas cada uma, onde foram construídos roteiros para cada uma das quatro fases do ciclo DSPD, cujos objetivos e fases são resumidos no Quadro 2.

Inicialmente, a pesquisadora apresentou o Termo de Consentimento Livre e Esclarecido aos que concordaram em participar da pesquisa. Logo após, realizou uma breve apresentação sobre como a Educação Permanente em Saúde se articula em um espaço de formação como o da residência multiprofissional em Saúde. Finalmente, foram definidos as normas e o funcionamento do grupo.

Cada reunião tinha objetivos específicos detalhados pela pesquisadora ao longo das fases da pesquisa (Quadro 2). A primeira reunião foi para a apresentação e recepção dos participantes. Os participantes da pesquisa se apresentaram e destacaram sua trajetória profissional. 
Quadro 2. Encontro, fase do Ciclo DSPD e objetivo do encontro.

\begin{tabular}{|l|l|l|}
\hline \multicolumn{1}{|c|}{ Reunião } & \multicolumn{1}{|c|}{ Fase } & \multicolumn{1}{c|}{ Objetivo } \\
\hline 1 & - & $\begin{array}{l}\text { Recepção e apresentação da metodologia } \\
\text { Entrega da primeira tarefa reflexiva }{ }^{1} \text { (Carta) }\end{array}$ \\
\hline 2 & Descoberta & $\begin{array}{l}\text { Apresentação da tarefa reflexiva e definição do tópico afirmativo } \\
\text { Entrega da segunda tarefa reflexiva (Registro das melhores práticas) }\end{array}$ \\
\hline 3 & Descoberta & $\begin{array}{l}\text { Apresentação da tarefa reflexiva } \\
\text { Insights, experiências positivas e melhores estratégias } \\
\text { Entrega da terceira tarefa reflexiva (Construindo os sonhos) }\end{array}$ \\
\hline 4 & Sonho & $\begin{array}{l}\text { Apresentação da terceira tarefa reflexiva } \\
\text { Imaginar a incorporação das estratégias } \\
\text { Entrega da quarta tarefa reflexiva (Revisão de literatura) }\end{array}$ \\
\hline 5 & Planejamento & $\begin{array}{l}\text { Apresentação da quarta tarefa reflexiva } \\
\text { Construção das proposições provocativas } \\
\text { Resumo das melhores estratégias a serem implementadas }\end{array}$ \\
\hline 6 & Destino & $\begin{array}{l}\text { Cronograma e plano de ação para implementar as estratégias definidas na RIMS } \\
{ }^{1} \text { Tarefa Reflexiva - atividade delegada para os participantes realizarem após a reunião, com apresentação do resultado no encontro posterior. }\end{array}$
\end{tabular}

Na primeira reunião, a pesquisadora apresentou e disponibilizou, aos preceptores, o estudo descrito por Souza ${ }^{11}$, destacando os pontos similares e diferentes da nossa proposta. Os participantes da pesquisa foram estimulados a aprofundarem seu conhecimento sobre a metodologia fazendo a leitura do texto, após o encontro. Ainda nessa reunião, a pesquisadora apresentou, aos participantes do estudo, as tarefas reflexivas. Nomeamos 'tarefas reflexivas' as atividades que os participantes realizavam anteriormente a cada reunião.

As tarefas reflexivas são atividades realizadas para possibilitar a reflexão acerca do objeto do estudo, funcionando como estratégia para preparar as reuniões do grupo. Essas atividades foram construídas pelas autoras, utilizando outros estudos de Pesquisa Apreciativa como modelo, e estão apresentadas no Quadro 3.

Quadro 3. Nome e questões orientadoras das tarefas reflexivas exploradas no ciclo DSPD.

\begin{tabular}{|l|l|l|}
\hline Tarefa Reflexiva & \multicolumn{1}{|c|}{ Nome } & \multicolumn{1}{c|}{ Questões orientadoras } \\
\hline 1 & Carta & $\begin{array}{l}\text { Descreva como foi realizar esta atividade? Quanto tempo levou? Como você se sentiu? } \\
\text { Do que você mais se orgulhou? Quais a opinião (aspectos positivos) dos seus colegas } \\
\text { sobre seu trabalho? }\end{array}$ \\
\hline 2 & $\begin{array}{l}\text { Registro das } \\
\text { melhores práticas }\end{array}$ & $\begin{array}{l}\text { Identifique duas práticas descritas que podem ser adotadas por todo o grupo e justifique } \\
\text { o porquê da decisão. }\end{array}$ \\
\hline 3 & $\begin{array}{l}\text { Construindo os } \\
\text { sonhos }\end{array}$ & $\begin{array}{l}\text { Descreva um sonho para a residência multiprofissional. Ou seja, se esta prática educativa } \\
\text { ainda não acontece na RIMS, como e de que forma ela poderia acontecer? }\end{array}$ \\
\hline 4 & $\begin{array}{l}\text { Revisão de } \\
\text { literatura }\end{array}$ & $\begin{array}{l}\text { Analise um artigo sobre a RIMS com experiências sobre atividades de ensino (papel do } \\
\text { preceptor) e atividades de EPS }\end{array}$ \\
\hline
\end{tabular}

Na primeira tarefa reflexiva, a qual antecedeu a fase da 'Descoberta', solicitamos que os participantes escrevessem uma carta - endereçada a algum colega, à coordenação de uma instituição ou para às pesquisadoras do estudo - sobre a trajetória profissional do preceptor, descrevendo uma atividade positiva realizada como preceptor na residência multiprofissional. Essa tarefa reflexiva foi considerada significativa pelos participantes, pois ressaltou elementos positivos em suas experiências enquanto preceptores. Além disso, destacaram que o exercício da escrita favoreceu um momento reflexivo raramente oportunizado no cotidiano de trabalho. 
A segunda e a terceira reuniões contemplaram a fase 'Descoberta', a primeira fase do ciclo DSPD. Essa fase pode ser iniciada com o diálogo, para encorajar os participantes da pesquisa a compartilharem experiências a fim de descobrirem ou redescobrirem os seus pontos fortes, ativos ou onde tiveram suas maiores conquistas ${ }^{9}$. É importante que se utilizem perguntas e ferramentas reflexivas que incentivem os participantes a lembrarem e contarem histórias sobre as suas experiências e a recordarem momentos em que tenham experimentado sucessos ${ }^{9}$.

A fase da Descoberta envolve, ainda, o descobrimento das melhores práticas dos participantes por meio da apreciação do que 'dá vida e energia' aos indivíduos, ao seu trabalho e à organização ${ }^{15}$. 0 compartilhamento de histórias positivas, a partir das experiências dos participantes, é uma estratégia eficaz para a produção de dados qualitativos significativos, conforme descrito por Carter ${ }^{7}$, Reed $^{6}$, Yoon $^{17}$. Nessa etapa, entrevistas feitas pela pesquisadora com os participantes, ou discussões em grupo, são as dinâmicas mais utilizadas 9 . No nosso estudo, tal fase foi realizada em duas reuniões. Nela os participantes identificaram e apreciaram experiências positivas que haviam realizado na residência multiprofissional enquanto preceptores. O primeiro objetivo da reunião dessa fase foi a apresentação da carta, por cada um dos participantes. Tal momento serviu para impulsionar o processo de descobrimento das melhores práticas dos preceptores e compartilhar com o grupo.

Após a apresentação da carta, o Tópico Afirmativo foi utilizado para guiar uma discussão em grupo'. O Tópico Afirmativo nessa reunião era: 'a residência multiprofissional é um espaço que possibilita a realização de práticas de Educação Permanente em Saúde', e, a partir desta afirmação, perguntou-se ao grupo: 'Quais as suas melhores práticas? Quais são as melhores práticas que esse grupo desenvolve? O que dá vida e energia no trabalho da residência multiprofissional? O que vocês consideram que esteja funcionando na residência multiprofissional?'

O objetivo da terceira reunião (a segunda da fase 'Descoberta') foi destacar e relacionar as melhores práticas apreciadas na reunião anterior. Para isso, os preceptores selecionaram e justificaram a sua decisão para o grupo. É importante salientar que as melhores práticas educativas foram escolhidas com base nos critérios de exequibilidade e abrangência dessas práticas, para que pudessem ser disseminadas por toda a RIMS. O consenso dos participantes foi construído pela relevância local e aplicabilidade para outras residências do Brasil.

Como tarefa reflexiva preparatória para a fase do 'Sonho', foi organizada a seguinte tarefa: 'escrever um sonho relacionado às práticas de ensino na residência multiprofissional e pensar em uma prática que ainda não acontece na residência multiprofissional em saúde'. Esta tarefa foi relatada pelos participantes como a mais significativa no processo, pois eles puderam pensar e trazer para a reunião os desejos que gostariam que se concretizassem.

A quarta reunião referiu-se à fase dos 'Sonhos'. Essa fase se configura quando as descobertas referentes às melhores práticas foram organizadas, especialmente quanto ao modo de implementar no futuro. A fase dos 'Sonhos' oferece, aos participantes, a oportunidade de explorarem coletivamente suas esperanças e desejos para o futuro da sua equipe ou organização'. Os participantes da pesquisa podem ser convidados a criar imagens positivas e atraentes da sua atuação profissional no futuro. Perguntas geradoras, conhecidas como 'questões milagrosas', são frequentemente utilizadas na Pesquisa Apreciativa, sendo os participantes convidados a visualizarem ou imaginarem como seria possível operacionalizar a proposta $20,27,30,31$.

Nesta pesquisa, a fase dos 'Sonhos' teve como objetivo imaginar a incorporação das práticas no cenário de atuação dos preceptores e, a partir disso, descrever as possíveis táticas que poderiam ser implementadas futuramente na residência multiprofissional. Neste momento, os preceptores foram convidados a imaginarem como poderia ser o futuro de sua atuação em uma residência multiprofissional com base no desenvolvimento de descrições. Porém, antes disso, cada participante apresentou uma síntese do seu sonho, de modo a compartilhar com o grupo suas ideias. Depois da apresentação dos sonhos, os preceptores realizaram um grupo de discussão sobre as questões que expressam as melhores formas possíveis de trabalhar as práticas de Educação Permanente em Saúde: 'Imagine se o que vocês consideram como melhores práticas se tornassem realidade institucional. Como elas acontecem? Qual seria a melhor forma de propagá-las? Quais as esperanças de isso se tornar realidade?' Essa atividade foi realizada por meio de uma discussão em grupo sobre as questões 
descritas acima. Como tarefa reflexiva, cada preceptor recebeu um artigo que tratava de educação permanente em cenários da formação, tal como nas residências multiprofissionais.

A penúltima fase, denominada 'Planejamento', foi iniciada na quinta reunião. Tal fase possibilita que os participantes criem estratégias para o planejamento das ações futuras na instituição, articulando um plano em que as pessoas envolvidas no estudo se sintam capazes de participar da concretização do sonho recentemente expressado, 15 .

A partir da fase de 'Planejamento', os participantes foram convidados a planejarem e registrarem as ações que devem ser implementadas. Esse momento exigiu empenho para fazer escolhas que possibilitassem atingir o futuro proposto?.

Nesse estudo, os preceptores construíram suas proposições provocativas por meio do confronto entre o que eles encontraram nos artigos disponibilizados pela pesquisadora e as suas experiências enquanto profissionais da residência. Tal fase foi dividida em três momentos, quando os participantes: 1) debateram o sonho coletivo, a partir dos modelos ou exemplos encontrados nos artigos, 2) desenvolveram as proposições provocativas "o que deve ser o ideal?" para o seu contexto, e discutiram quais delas teriam a possibilidade de ser operacionalizadas, e 3) desenharam as possíveis ações para serem implementadas futuramente, construindo um quadro com os melhores exemplos encontrados, a partir das atividades realizadas.

A fase 'Destino' é a última fase do ciclo DSPD, e foi iniciada na sexta reunião. Nesse momento, foram discutidas as possibilidades de implementar os planos construídos na fase anterior. Essa fase também é utilizada para organizar projetos e priorizar iniciativas em direção à discussão e implementação de projetos futuros ${ }^{27}$. O grupo pode desenvolver um cronograma de atividades, estratégias de comunicação e uma lista de medidas de acompanhamento do impacto dos esforços do projeto ${ }^{9}$.

Nessa última fase, a pesquisadora iniciou a discussão com os participantes orientada pelas seguintes questões: 'Como implementar esta estratégia? Como capacitar, aprender e improvisar para as melhores práticas? Como compartilhar estes resultados?'

A partir de tais questões, foram construídos, juntamente com os participantes, um cronograma e um plano de ação que ajudassem os preceptores a implementarem, na RIMS, a estratégia escolhida pelos participantes. A implementação encontra-se em andamento, tendo ocorrido um encontro adicional entre a pesquisadora e os participantes para tratarem do processo de operacionalização da estratégia.

\section{Pontos positivos e desafios da metodologia}

Como ressaltado anteriormente, a Pesquisa Apreciativa apresenta uma abordagem metodológica inovadora no campo da pesquisa qualitativa. Dois pontos positivos se destacam: o aprendizado coletivo para propor melhoras às organizações e a criação de produtos de interesse para os participantes.

A Pesquisa Apreciativa é inclusiva, colaborativa, e sua capacidade para estimular a aprendizagem coletiva deve ser destacada. Trajkovski ${ }^{10}$ dizem que a Pesquisa Apreciativa permite que os membros possam compartilhar o conhecimento tácito e explícito necessário para construir um planejamento das ações desejados para a sua organização. No nosso estudo, tendo em consideração o grau de formação dos participantes, propusemos a criação de uma produção científica coletiva, aceita pelos participantes da pesquisa que serão coautores de um artigo. É importante mencionar que, em outras metodologias qualitativas participativas, os participantes do estudo também "podem ser convidados a se incluir na equipe de pesquisa, a partilhar das reflexões e resultados e a serem coautores nas publicações" 32 (p. 773).

Atualmente, no Brasil, os profissionais de saúde têm dificuldade para participar de pesquisas, sobretudo devido à indisponibilidade de tempo e carência de benefícios aos participantes. Diante deste cenário e reconhecendo a contribuição intelectual das pessoas que se dispuseram a participar, a possibilidade de produção científica coletiva foi importante para que pudéssemos recrutar participantes, já que os mesmos a necessitam para sua progressão profissional. Tal estratégia é 
pertinente no contexto deste estudo, mas constitui uma opção. Assim, destacamos que a metodologia da Pesquisa Apreciativa possibilita que os participantes se engajem e determinem a orientação do projeto por meio de reflexões conjuntas para o desenvolvimento de estratégias de mudança nos processos.

Por fim, destacamos dois desafios da Pesquisa Apreciativa que são a centralidade da capacidade da pesquisadora para responder às diferentes propostas dos participantes em cada fase e a questão ética de identificação dos participantes do estudo, por intermédio de atividades desenvolvidas nos serviços, o que inclui a publicação dos resultados. Quando nos referimos à centralidade da capacidade da pesquisadora, apresentamos sugestões para auxiliar na condução da pesquisa, as quais podem ser: conhecer a metodologia em profundidade, manter o grupo no caminho apreciativo e evitar a tendência de ver apenas os problemas nas situações vivenciadas, coordenar as reuniões objetivas e no tempo previsto, mas, ao mesmo tempo, fazê-las reflexivas, dar respostas às perspectivas dos participantes tendo capacidade de improvisação mas mantendo a orientação da metodologia. Em termos éticos, é necessário discutir com os participantes o impacto individual e coletivo de sua identificação. Os mesmos devem consentir cada forma de identificação, por exemplo: que as publicações levem o seu nome, que os outros profissionais saibam que participam do estudo, que a coordenação do seu serviço saiba, etc.

\section{Considerações finais}

Em resumo, a Pesquisa Apreciativa é uma metodologia que permite a participação e o engajamento de profissionais da saúde em pesquisas relacionadas a sua área de atuação, com potencial para ser aplicada em múltiplas áreas. Além disso, observamos que a Pesquisa Apreciativa incentiva os debates reflexivos e críticos por parte dos participantes, estabelecendo um espaço de discussão para que as mudanças ocorram.

Neste artigo, apresentamos o detalhamento da construção metodológica de uma Pesquisa Apreciativa em particular. Queremos destacar que os profissionais da saúde aprovaram o encorajamento para adotarem uma abordagem positiva, construtiva e dialógica para propor mudanças no coletivo da instituição da qual fazem parte. O trabalho de campo revelou uma adesão significativa à metodologia por parte dos participantes da pesquisa. Salientamos que a possibilidade da construção de uma produção científica com os participantes da pesquisa constitui uma inovação no cenário das pesquisas em saúde no Brasil.

Outro aspecto importante foi a boa qualidade dos dados produzidos nos momentos de reflexão e debates sobre questões críticas em relação às práticas de Educação Permanente em Saúde na RIMS. Outra contribuição também foi estabelecer um espaço de discussão sobre as mudanças necessárias para promover avanços no programa da RIMS, nos serviços de saúde e na formação, o que põe em evidência o potencial da Pesquisa Apreciativa.

\section{Colaboradores}

Cristiane Trivisiol Arnemann participou ativamente da discussão dos resultados e da revisão e da aprovação da versão final do trabalho. Denise Gastaldo participou ativamente da discussão dos resultados e da revisão e da aprovação da versão final do trabalho. Maria Henriqueta Kruse participou ativamente da discussão dos resultados e da revisão e da aprovação da versão final do trabalho. 


\section{Referências}

1. Young L. Participatory action research (chapter 24). In: Beck CT, editor. International handbook of qualitative nursing research. New York: Routledge; 2013. p. 319-30.

2. Gastaldo D, Magalhães L, Carrasco C, Davy C. Body-map storytelling as research: methodological considerations for telling the stories of undocumented workers through body mapping [Internet]. 2012 [citado 16 Jan 2016]. Disponível em: http://www. migrationhealth.ca/undocumented-workers-ontario/body-mapping.

3. Gooden A, Gastaldo D. Partnerships for participatory action research: the case of recent immigrant women in Toronto, Canada. The collaborative turn: working together in qualitative research. Netherlands: Sense Publisher; 2009. p. 71-88.

4. Zaforteza C, Gastaldo D, Moreno C, Bover A, Miró R, Miró M. Transforming a conservative clinical setting: ICU nurses' strategies to improve care for patients' relatives through a participatory action research. Nurs Inq. 2015; 22(4):336-47.

5. Cooperrider D. Appreciative inquiry: toward a methodology for understanding and enhancing organizational innovation [dissertation]. Cleveland: Western Reserve University; 1986.

6. Reed J. Appreciative inquiry: research for change. London: Sage Publications; 2007.

7. Carter B. 'One expertise among many' - working appreciatively to make miracles instead of finding problems: using appreciative inquiry to reframe research. J Res Nurs. 2006; 11(1):48-63.

8. Kavanagh PM. Appreciative inquiry: an interactive organizational intervention to translate acute pain management evidence into pediatric nursing practice [thesis]. Toronto: University of Toronto; 2010.

9. Trajkovski S, Schmied V, Vickers M, Jackson D. Implementing the 4D cycle of appreciative inquiry in health care: a methodological review. J Adv Nurs. 2012; 69(6):1224-34.

10. Trajkovski S, Schmied V, Vickers MH, Jackson D. Experiences of neonatal nurses and parents working collaboratively to enhance family centred care: the destiny phase of an appreciative inquiry project. Collegian. Forthcoming 2016.

11. Souza VL, McNamee $S$, Santos MA. Avaliação como construção social: investigação apreciativa. Psicol Soc. 2010; 22(3):598-607.

12. Jorge JS, Corradi-Webster CM. Consultório de rua: contribuições e desafios de uma prática em construção. Saude Transform Soc. 2012; 3(1):39-48.

13. Nilson LG, Campos DA, Dallegrave EJ, Moretti-Pires RO. A investigação apreciativa como tecnologia para a pesquisa em saúde coletiva. Saude Transform Soc. 2014; 5(3):1-9.

14. Poland B, Cavalcante Jr F. Unmasking power relations: from interview research to dialogue for social change. CQ seminar series [Internet]. [citado 16 Jan 2016]. Disponível em: http://www. migrationhealth.ca/undocumented-workers-ontario/body-mapping.

15. Cooperrider D, Whitney D, Stavros JM. Appreciative inquiry handbook: the first in a series of ai workbooks for leaders of change. Ohio: Crown Custom Publishing Inc; 2005.

16. Coghlan AT, Preskill H, Tazavaras Catsambus T. An overview of appreciative inquiry in evaluation. New Dir Eval. 2003; 100:5-18.

17. Yoon $M N$, Lowe $M$, Budgell $M$, Steele $C M$. An exploratory investigation using appreciative inquiry to promote nursing oral care. Geriatr Nurs. 2011; 30(5):326-40.

18. Lind C, Smith D. Analyzing the state of community health nursing. Adv Nurs Sci. 2008; 31(1):28-41. 
19. Havens DS, Wood SO, Leeman J. Improving nursing practice and patient care: building capacity with appreciative inquiry. J Nurs Admin. 2006; 36(10):463-70.

20. Wright $M$, Baker $A$. The effects of appreciative inquiry interviews on staff in the UK National Health Service. Int J Health Care Qual Assur Inc Leadersh Health Serv. 2005; 18(1):41-61.

21. Ruhe MC, Bobiak SN, Litaker D, Carter CA, Wu L, Schroeder C, et al. Appreciative inquiry for quality improvement in primary care practices. Qual Manag Health Care. 2011; 20(1):37-48.

22. Scerri A, Innes A, Scerri C. Discovering what works well: exploring quality dementia care in hospital wards using an appreciative inquiry approach. J Clin Nurs. 2015; 24(13-14):1916-25.

23. Jongudomkarn D, Macduff C. Development of a family nursing model for prevention of cancer and other noncommunicable diseases through an appreciative inquiry. Nurs Sci Q. 2014; 27(23):315-23.

24. Falk KJ. Appreciative inquiry with nurses who work with children of incarcerated parents. Nurs Sci Q. 2014; 27(4):315-23.

25. Baker $A$, Wright $M$. Using appreciative inquiry to initiate a managed clinical network for children's liver disease in the UK. Int J Qual Health Care. 2006; 19(6-7):561-74.

26. Lazic J, Radenovic M, Arnfield A, Janic D. Implementation of a nurse education programme in pediatric oncology using appreciative inquiry: a single center experience in Belgrade, Serbia. Eur J Oncol Nurs. 2011; 15(5):524-7.

27. Shendell-Falik N, Feinson M, Mohr BJ. Enhancing patient safety: improving the patient handoff process through appreciative inquiry. J Nurs Adm. 2007; 37(2):95-104.

28. Bonham E. Appreciative inquiry in youthful offender psychiatric nursing research. J Child Adolesc Psychiatr Nurs. 2011; 24(2):122-9.

29. Knibbs K, Underwood J, MacDonald M, Schoenfeld B, Lavoie-Tremblay $M$, Crea-Arsenio $M$, et al. Appreciative inquiry: a strength-based research approach to building Canadian public health nursing capacity. J Res Nurs. 2010; 17(5):484-94.

30. Reed J, Pearson P, Douglas B, Swinburne S, Wilding H. Going home from hospital: an appreciative inquiry study. Health Soc Care Commun. 2002; 10(1):36-45.

31. Carter B, Cummings J, Cooper L. An exploration of best practice in multi-agency working and the experiences of families of children with complex health needs: what works well and what needs to be done to improve practice for the future? J Clin Nurs. 2007; 16(3) :527-539.

32. Guerriero ICZ, Minayo MCS. O desafio de revisar aspectos éticos das pesquisas em ciências sociais e humanas: a necessidade de diretrizes específicas. Physis. 2013; 23(3):763-82. 
Arnemann CT, Gastaldo D, Kruse MHL. Investigación Apreciativa: características, utilización y posibilidades para el área de la Salud en Brasil. Interface (Botucatu). 2018; 22(64):121-31.

Nuestro artículo presenta y discute las características de la Investigación Apreciativa como metodología de investigación, utilizando un ejemplo de una residencia multiprofesional integrada en Salud en Brasil. La Investigación Apreciativa, conocida en inglés como Appreciative Inquiry, es una metodología usada para identificar las mejores prácticas desarrolladas y empleadas por las personas que trabajan en una institución. Esa metodología permite la participación y el compromiso de profesionales del área de la Salud en investigaciones relacionadas a su área de actuación, con potencial de aplicación en múltiples áreas. Además, la Investigación Apreciativa incentiva debates reflexivos y críticos por parte de los participantes, estableciendo un espacio de discusión para que ocurran cambios. Los profesionales de la Salud que participaron en ese trabajo consideran pertinente la finalidad de alentar a las personas para que adopten un abordaje positivo, constructivo y dialógico para proponer cambios institucionales.

Palabras clave: Investigación Apreciativa. Investigación cualitativa. Métodos. Metodología. Práctica profesional. 
